\title{
ACKNOWLEDGMENT
}

The authors would like to thank L. Wilhoit for his assistance in analyzing the data.

*Research supported by the U. S. Atomic Energy Commission.

$\dagger$ Present address: Department of Chemistry, Columbia University, New York, New York 10027.

${ }^{1}$ J. E. Kitching, Bull. Am. Phys. Soc. 17, 578 (1972); and to be published.

${ }^{2}$ R. D. Lawson, private communication.

${ }^{3} \mathrm{H}$. Ohnuma and J. L. Yntema, Phys. Rev. 178, 1855 (1969).

${ }^{4}$ G. Bassani and J. Picard, Nucl. Phys. A131, 653 (1969).

${ }^{5}$ J. B. Ball and C. B. Fulmer, Phys. Rev. 172, 1199 (1968).

${ }^{6}$ R. L. Kozub and D. H. Youngblood, Phys. Rev. Letters 28, 1529 (1972); following paper, Phys. Rev. C $\underline{7}$, 410 (1973).

'R. L. Kozub and D. H. Youngblood, Phys. Rev. C $\underline{4}$,
535 (1971).

${ }^{8}$ Received from P. D. Kunz, University of Colorado.

${ }^{9}$ F. D. Becchetti, Jr., and G. W. Greenlees, Phys. Rev. 182, 1190 (1969).

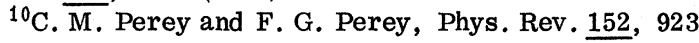
(1966).

${ }^{11}$ E. R. Flynn, D. D. Armstrong, J. G. Berry, and

A. G. Blair, Phys. Rev. 182, 1113 (1969).

${ }^{12}$ W. H. Smith, Phys. Rev. Letters 23 , 1045 (1969); and private communication.

${ }^{13}$ W. J. Thompson and W. R. Hering, Phys. Rev. Letters $24,272(1970)$.

${ }^{14} \mathrm{~A} . \overline{\mathrm{H}}$. Wapstra and N. B. Gove, Nucl. Data $\underline{\mathrm{A9}}, 267$ (1971).

${ }^{15} \mathrm{~J}$. V. Maher, J. R. Comfort, and G. C. Morrison, Phys. Rev. C $\underline{3}, 1162$ (1971).

\section{Study of Hole-State Analogs in Mo Isotopes*}

\author{
R. L. Kozub† and D. H. Youngblood \\ Cyclotron Institute, Texas A\&M University, College Station, Texas 77843
}

(Received 9 June 1972)

\begin{abstract}
A study of isobaric-analog states (IAS) excited in the $(p, d)$ and $(d, t)$ reactions on ${ }^{92,}{ }^{94,}{ }^{96} \mathrm{Mo}$, the $\left({ }^{3} \mathrm{He}, \alpha\right)$ reaction on ${ }^{92,}{ }^{96} \mathrm{Mo}$, and the ${ }^{98} \mathrm{Mo}(p, d){ }^{97} \mathrm{Mo}$ reaction is reported. Coulomb energies and angular distributions were measured for analogs of all parent states observed in a $\left(d,{ }^{3} \mathrm{He}\right)$ study on Mo targets, and some additional nuclear structure information was obtained. Distorted-wave Born-approximation calculations were performed, and the resulting spectroscopic factors reveal a pronounced decrease in $l=1$ hole strength with increasing mass number as well as fluctuations in the ratios $C^{2} S_{n}(p, d) / C^{2} S_{n}(d, t)$. The first effect is discussed in terms of mixing with the dense spectrum of $T_{<}$levels in the IAS region.
\end{abstract}

\section{INTRODUCTION}

Isobaric-analog states (IAS) in the $A=90$ region have been studied extensively via proton resonances ${ }^{1}$ and, more recently, in proton stripping reactions. ${ }^{2-4}$ However, no extensive studies of $T$, hole states have been reported for this region, which probably reflects the fact that relatively high bombarding energies are required to obtain meaningful direct-reaction data for the IAS. In the present work, IAS excited in the $(p, d)$ and $(d, t)$ reactions on ${ }^{92,94,96} \mathrm{Mo}$ and the ${ }^{98} \mathrm{Mo}(p, d)^{97} \mathrm{Mo}$ reaction were investigated. Differential cross-section measurements were also obtained at a few angles for the $\left({ }^{3} \mathrm{He}, \alpha\right)$ reaction on ${ }^{92,96} \mathrm{Mo}$. A comparison of spectroscopic strengths deduced from these reactions is presented, with further comparisons to the parent state results of Ohnuma and Yntema $(\mathrm{OY})^{5}$ who studied the $\left(d,{ }^{3} \mathrm{He}\right)$ reaction on the molybdenum isotopes. A brief account of this work has been reported elsewhere. ${ }^{6}$

\section{EXPERIMENTAL PROCEDURE}

The experimental configuration and data analysis procedure was similar to that reported previously. ${ }^{4}$ Data were taken with $38.6-\mathrm{MeV}$ protons, $40.6-\mathrm{MeV}$ deuterons, and $35.0-\mathrm{MeV}$ helions incident on isotopically enriched Mo targets whose weighed thicknesses ranged from 0.554 to $1.10 \mathrm{mg} / \mathrm{cm}^{2}$. Two 


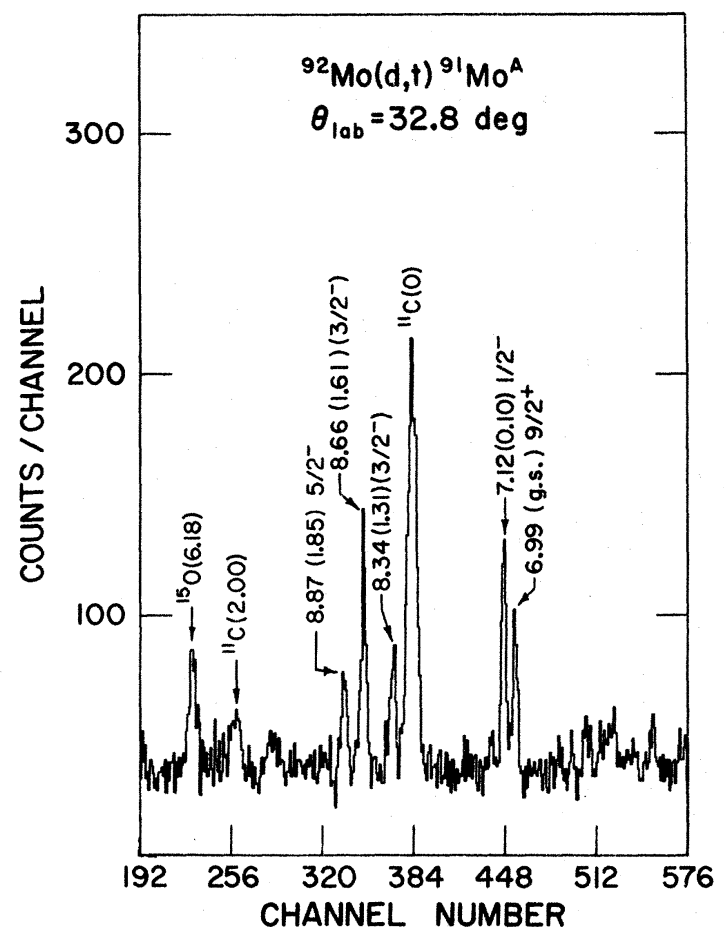

FIG. 1. Spectrum for the ${ }^{92} \mathrm{Mo}(d, t)^{91} \mathrm{Mo}^{A}$ reaction at $\theta_{\text {lab }}=32.8^{\circ}$. Excitation energies are in $\mathrm{MeV}$. Values in parentheses for Mo levels are energies of corresponding $\mathrm{Nb}$ parent states.

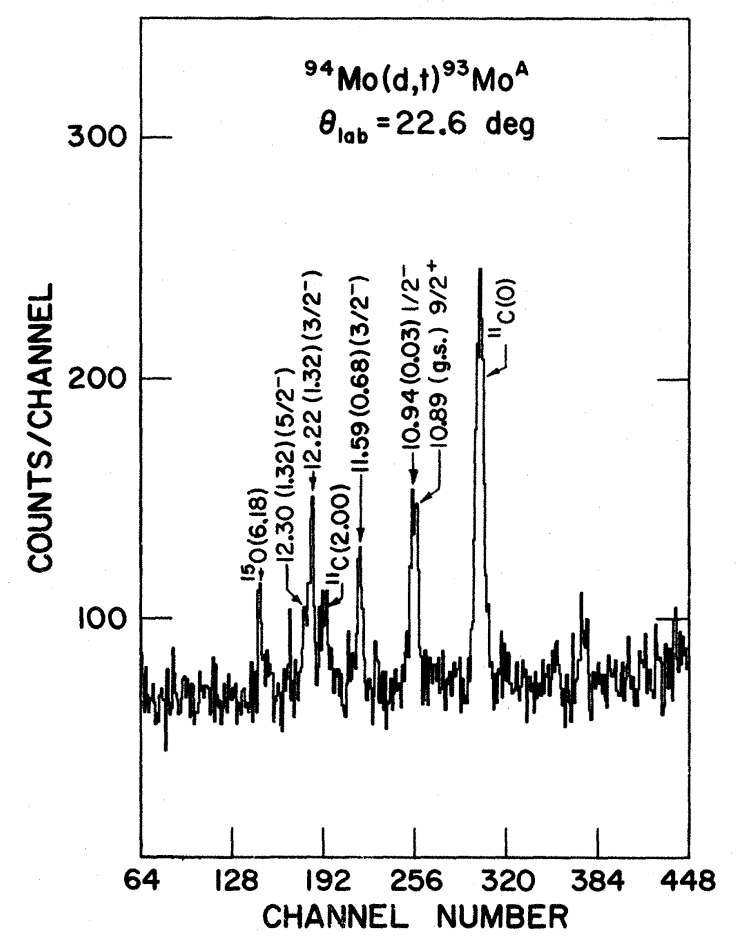

FIG. 2. Spectrum for the ${ }^{94} \mathrm{Mo}(d, t){ }^{93} \mathrm{Mo}^{A}$ reaction at $\theta_{\text {lab }}=22.6^{\circ}$. See also Fig. 1 caption.

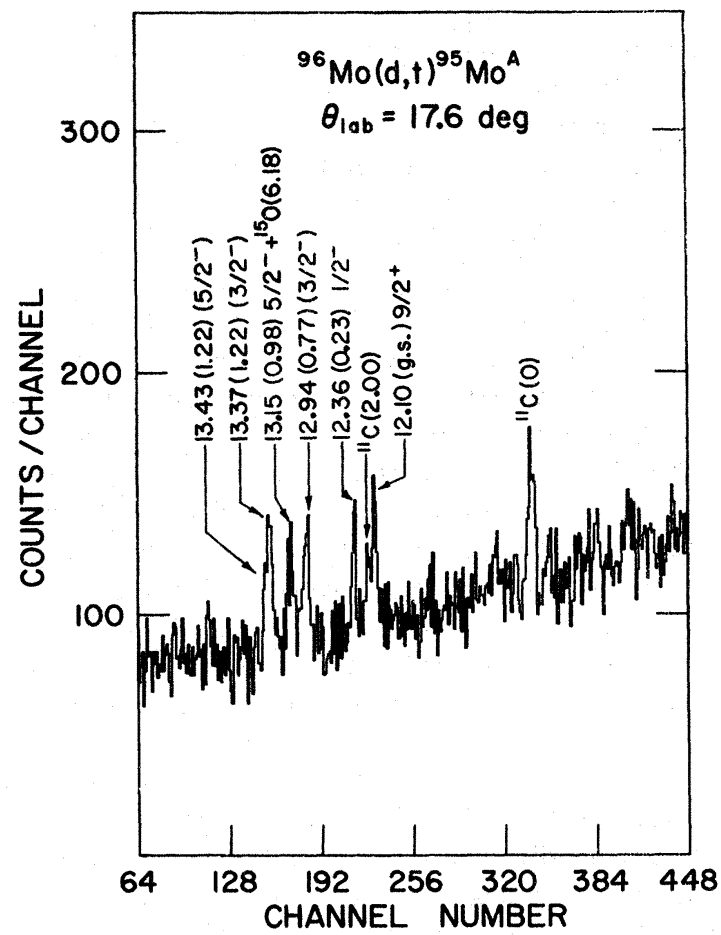

FIG. 3. Spectrum for the ${ }^{96} \mathrm{Mo}(d, t){ }^{95} \mathrm{Mo}^{A}$ reaction at $\theta_{\mathrm{lab}}=17.6^{\circ}$. See also Fig. 1 caption.

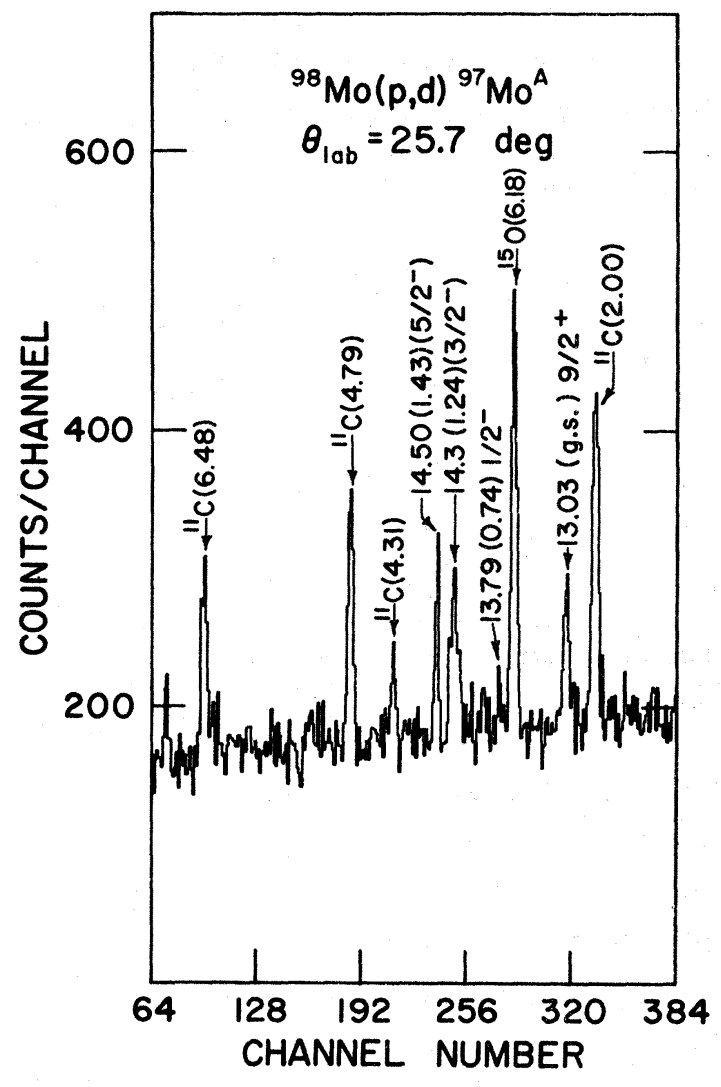

FIG. 4. Spectrum for the ${ }^{98} \mathrm{Mo}(p, d)^{97} \mathrm{Mo}^{A}$ reaction at $\theta_{\text {lab }}=25.7^{\circ}$. See also Fig. 1 caption. 
counter telescopes spaced $5^{\circ}$ apart were used simultaneously to reduce data acquisition time, and selected data points were checked by measurement with both systems. For the $(p, d)$ and $(d, t)$ reactions, each telescope consisted of a surfacebarrier detector in the thickness range 0.5-1.0 $\mathrm{mm}$ for $\Delta E$, a $3-\mathrm{mm} \mathrm{Si}(\mathrm{Li})$ detector for $E$, and a third detector in anticoincidence with $\Delta E$ and $E$ to eliminate pulses due to elastically scattered particles. The over-all resolution obtained was about $50 \mathrm{keV}$ full width at half maximum. A peakfitting computer code was used to extract yields for partially resolved levels. Spectra in the IAS region for the $(d, t)$ reaction on ${ }^{92,94,96} \mathrm{Mo}$ and the ${ }^{98} \mathrm{Mo}(p, d){ }^{97} \mathrm{Mo}$ reaction are shown in Figs. $1-4$.

Energy calibrations were obtained from neutronpickup reactions on ${ }^{60} \mathrm{Ni},{ }^{94,96,98} \mathrm{Mo}$, and the ${ }^{12} \mathrm{C}$ and ${ }^{16} \mathrm{O}$ contaminants present in the targets. Absolute cross-section normalizations were obtained by integrating the beam current and checked by measuring proton elastic scattering cross sections at forward angles. Excellent agreement with optical-model predictions ( $\$ 5 \%$ ) was obtained in all cases, and the maximum disagreement among experimental values from the targets of different $A$ was $\approx 5 \%$ at $\theta_{\text {c.m. }}=7.8^{\circ}$.

\section{DISTORTED-WAVE BORN- APPROXIMATION CALCULATIONS}

Distorted-wave Born-approximation (DWBA) calculations, including finite range and nonlocal (FRNL) corrections were performed using the computer code DWUCK ${ }^{7}$ and optical-model parameters from the literature..$^{4-11}$ These parameters and the FRNL parameters are listed in Table I. The same deuteron, triton, helion, and $\alpha$ parameters were used for all calculations, while the proton parameters were adjusted slightly as a function of target mass and isospin according to the prescription of Ref. 8. Neutron form factors were calculated for potentials of radius $1.23 \mathrm{~A}^{1 / 3}$ $\mathrm{fm}$, diffuseness $0.65 \mathrm{fm}$, and $\lambda_{\mathrm{so}}=25$, with binding energies equal to the experimental separation energies. These wave functions reproduce the proton form factors used by $\mathrm{OY}^{5}$ for the corresponding parent states to within $2 \%$ in the nuclear interior for all levels considered here. (The potentials used by OY had $R=1.20 A^{1 / 3} \mathrm{fm}, a=0.65 \mathrm{fm}$, and $\lambda_{\text {so }}=25$.)

The calculated and experimental cross sections are related by

$$
\left(\frac{d \sigma}{d \Omega}\right)_{\exp }=\frac{N C^{2} S}{2 J+1}\left(\frac{d \sigma}{d \Omega}\right)_{\text {DwUCK }}
$$

where $J$ is the transferred angular momentum, $N$ is the normalization constant determined from the internal structure of the projectiles, and $C^{2} S$ is the spectroscopic factor. Values of $N$ were taken from $\operatorname{Smith}^{12}$ for the $(p, d)$ reaction (2.54) and from Thompson and Hering ${ }^{13}$ for the $(d, t)$ reaction (also 2.54). In order to provide a more meaningful comparison of absolute spectroscopic factors, the FRNL $\left(d,{ }^{3} \mathrm{He}\right)$ results of Ref. 5 were adjusted to correspond to $N=2.30$, which was also calcu-

TABLE I. Optical-model and FRNL parameters used in DWBA calculations (MeV fm units).

\begin{tabular}{|c|c|c|c|c|c|c|c|c|c|c|c|}
\hline Particle & $V$ & $r_{0}$ & $a$ & $W$ & $4 W_{D}$ & $r_{I}$ & $a_{I}$ & $V_{\text {so }}$ & $r_{\text {so }}$ & $\beta^{2 \mathrm{a}}$ & $R^{\mathrm{b}}$ \\
\hline$-p^{\mathrm{c}}$ & 47.4 & 1.170 & 0.750 & 5.84 & 12.56 & 1.320 & 0.570 & 6.20 & 1.010 & 0.85 & \multirow{3}{*}{$\begin{array}{l}0.695 \\
0.845\end{array}$} \\
\hline$d \mathrm{~d}$ & 100.8 & 1.099 & 0.835 & & 53.64 & 1.344 & 0.747 & 6.53 & 1.099 & 0.54 & \\
\hline$t^{\mathrm{e}}$ & 151.1 & 1.240 & 0.685 & 24.06 & & 1.432 & 0.870 & & & 0.25 & \\
\hline${ }^{3} \mathrm{He}^{\mathrm{f}}$ & 157.8 & 1.174 & 0.706 & 11.71 & & 1.596 & 1.032 & & & 0.25 & \multirow{3}{*}{2.00} \\
\hline$\alpha^{g}$ & 228.0 & 1.366 & 0.577 & 23.30 & & 1.242 & 0.577 & & & 0.20 & \\
\hline$n$ & & 1.23 & 0.65 & & & & & $\lambda_{\mathrm{so}}=25$ & & 0.85 & \\
\hline
\end{tabular}

a Nonlocal parameter used in DWUCK.

${ }^{\mathrm{b}}$ Finite-range parameter for $(p, d),(d, t)$, and $\left({ }^{3} \mathrm{He}, \alpha\right)$ reactions, respectively, used in DWUCK.

${ }^{c}$ Proton parameters were obtained from the equations of Ref. 8:

$$
\begin{aligned}
& V=54-0.32 E+0.4 Z A^{-1 / 3}+24(N-Z) / A, \\
& W=0.22 E-2.7, \\
& W_{D}=11.8-0.25 E+12(N-Z) / A, \\
& a_{I}=0.51+0.7(N-Z) / A, \text { with } \\
& \quad r_{0}, a, r_{I}, V_{\text {so }}, r_{\text {so }}, \text { and } a_{\text {so }}=a \text { fixed. Parameters shown in table are for }{ }^{92} \text { Mo. } \\
& \text { d See Ref. } 9 . \\
& \text { e See Ref. } 10 . \\
& \text { f See Ref. } 4 . \\
& \text { g See Ref. } 11 .
\end{aligned}
$$


lated by Ref. 13. We consider only relative spectroscopic factors for the $\left({ }^{3} \mathrm{He}, \alpha\right)$ reaction.

\section{RESULTS AND DISCUSSION}

A. Nuclear Structure

Analogs corresponding to all levels seen in the $\left(d,{ }^{3} \mathrm{He}\right)$ work of Ref. 5 were observed in the present work (Figs. 1-4). The proton-hole structure is very similar for all isotopes, with most of the $1 g_{9 / 2}$ and $2 p_{1 / 2}$ strength contained in a single transition from each of these orbits, while most of the $2 p_{3 / 2}$ strength appears to be in two levels of roughly comparable strength. A sizable fraction of the $1 f_{5 / 2}$ strength is also observed.

Angular distributions for IAS excited in the $(p, d)$ and $(d, t)$ reactions are shown in Figs. 5-9 along with DWBA predictions. The DWBA fits to the data for the $(d, t)$ reaction are quite good, while the $(p, d)$ curves are generally in agreement with the data in the vicinity of forward maxima. The $J^{\pi}$ values assigned to the parent states by $O Y^{5}$ are consistent with our results for the IAS. The better resolution of the IAS experiment has permitted
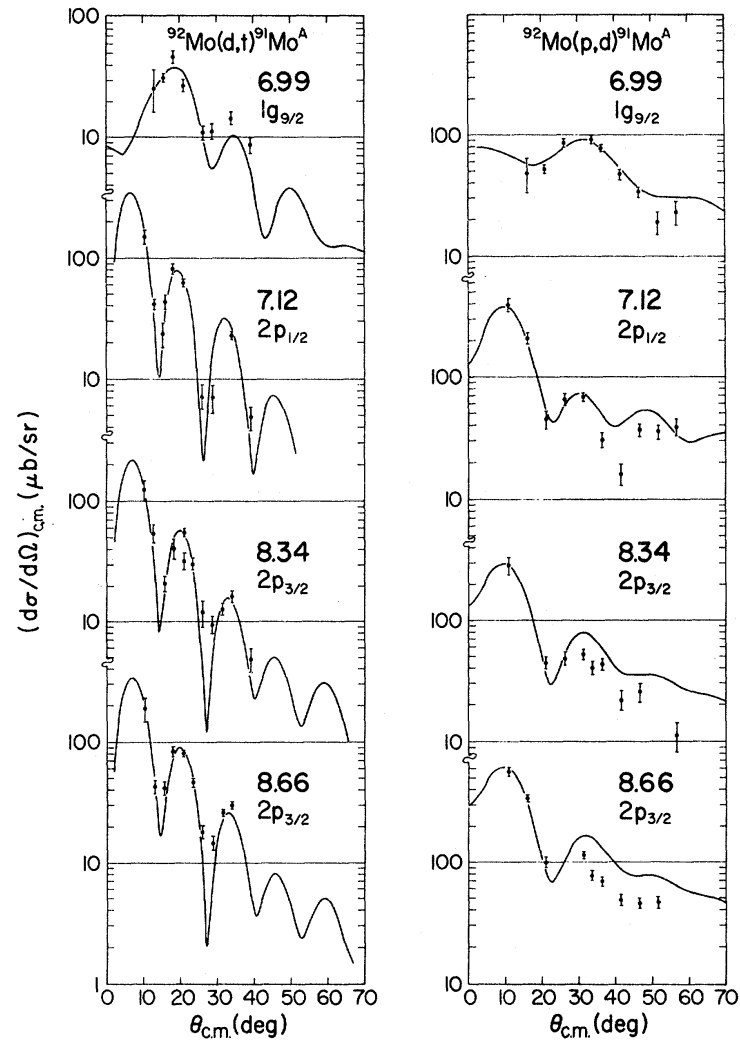

FIG. 5. Angular distributions for $1 g$ and $2 p$ IAS excited in the $(d, t)$ and $(p, d)$ reactions on ${ }^{92}$ Mo. Excitation energies are in $\mathrm{MeV}$, and the curves are DWBA predictions. decomposition of the analogs of four multiplets observed by Ref. 5. A mixture of states at $1.22 \mathrm{MeV}$ excitation in ${ }^{95} \mathrm{Nb}$ containing some $l=1$ strength was reported by OY. We have extracted angular distributions for two levels from the analog of this group (13.4 MeV in ${ }^{95} \mathrm{Mo}$ ) (Fig. 3), one of which is due to an $l=1$ transfer (bottom curves in Fig. 7). The data for the other component are not conclusive, although an $l=3$ calculation is consistent with the data from both reactions (bottom curves in Fig. 8). The group at $14.3 \mathrm{MeV}$ in ${ }^{97} \mathrm{Mo}$ (Fig. 4) was fitted consistently with four peaks at excitation energies $14.26,14.30,14.34$, and 14.38 MeV. Meaningful angular distributions were obtained for only the 14.30- and 14.34-MeV levels, both of which are $l=1$ (Fig. 9), in agreement with the $J^{\pi}$ of $\left(\frac{3}{2}^{-}\right)$assigned to the entire parent state group by $O Y$. Individual angular distributions were also obtained for the IAS in ${ }^{93} \mathrm{Mo}$ corresponding to the ground-state and $1.32-\mathrm{MeV}$ doublets ${ }^{3}$ in ${ }^{93} \mathrm{Nb}$. The observed widths of the IAS groups are limited by the experimental resolution within statistics except for the $\frac{9^{+}}{2}$ IAS in ${ }^{97} \mathrm{Mo}$, for which we
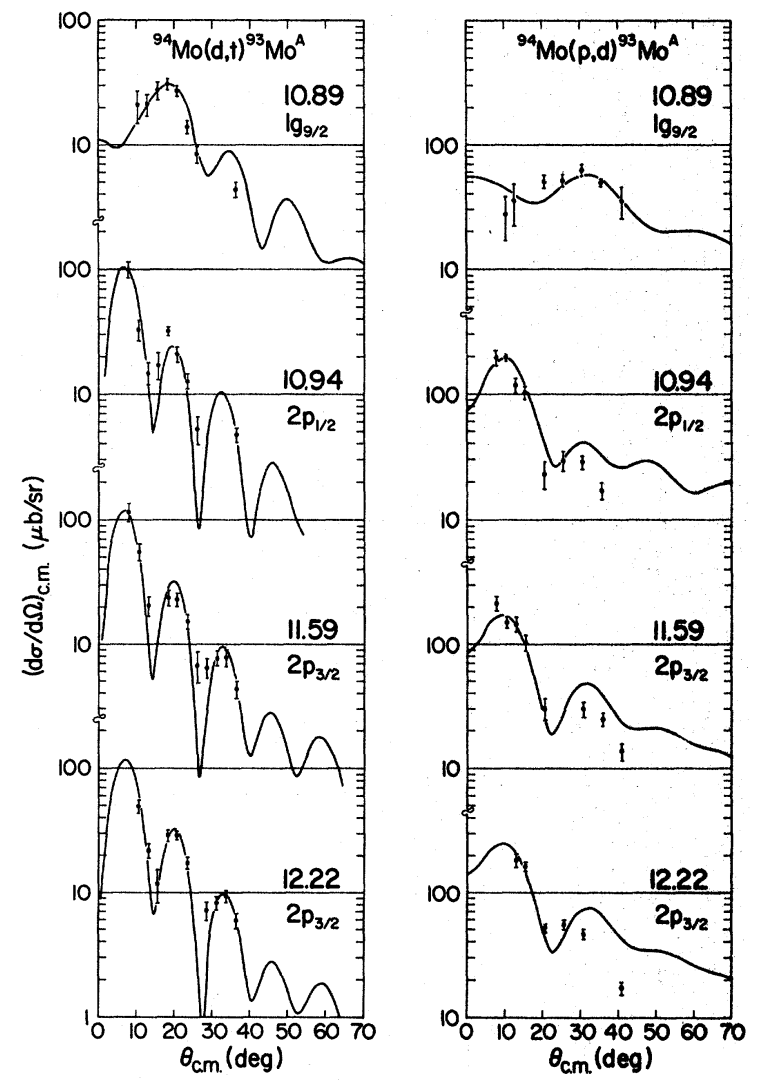

FIG. 6. Angular distributions for $1 g$ and $2 p$ IAS excited in the $(d, t)$ and $(p, d)$ reactions on ${ }^{94} \mathrm{Mo}$. Excitation energies are in MeV, and the curves are DWBA predictions. 
obtain a natural width, $\Gamma=70 \pm 20 \mathrm{keV}$. A summary of experimental results is given in Table II.

\section{B. Coulomb Energies}

The Coulomb energies listed in Table II were calculated from the relation ${ }^{14}$

$$
\Delta_{C}=M_{Z+1}^{A *}-M_{Z}^{A *}+\Delta_{n p},
$$

where $\Delta_{n p}=0.7824 \mathrm{MeV}$ is the neutron-hydrogen atomic-mass difference and $Z=41$ in calculations for the Nb-Mo isobars. Mass values for $A \geqslant 93$ are those of Mattauch, Thiele, and Wapstra, ${ }^{15}$ while values obtained from more recent measurements $^{16,17}$ were used for $A=91$. Niobium excitation energies were obtained from Ohnuma and Yntema ${ }^{5}$ and Hesse and Finckh. ${ }^{18}$ Our values for $\Delta_{c}$ are in good agreement with measurements for other nuclei in this mass region ${ }^{14}$ and decrease slowly with increasing neutron excess. The absolute errors in $\Delta_{c}$ are $\pm 30-50 \mathrm{keV}$, whereas the relative errors for a given $A$ (i.e., the errors in level spacings) are about $\pm 10 \mathrm{keV}$ in most cases.
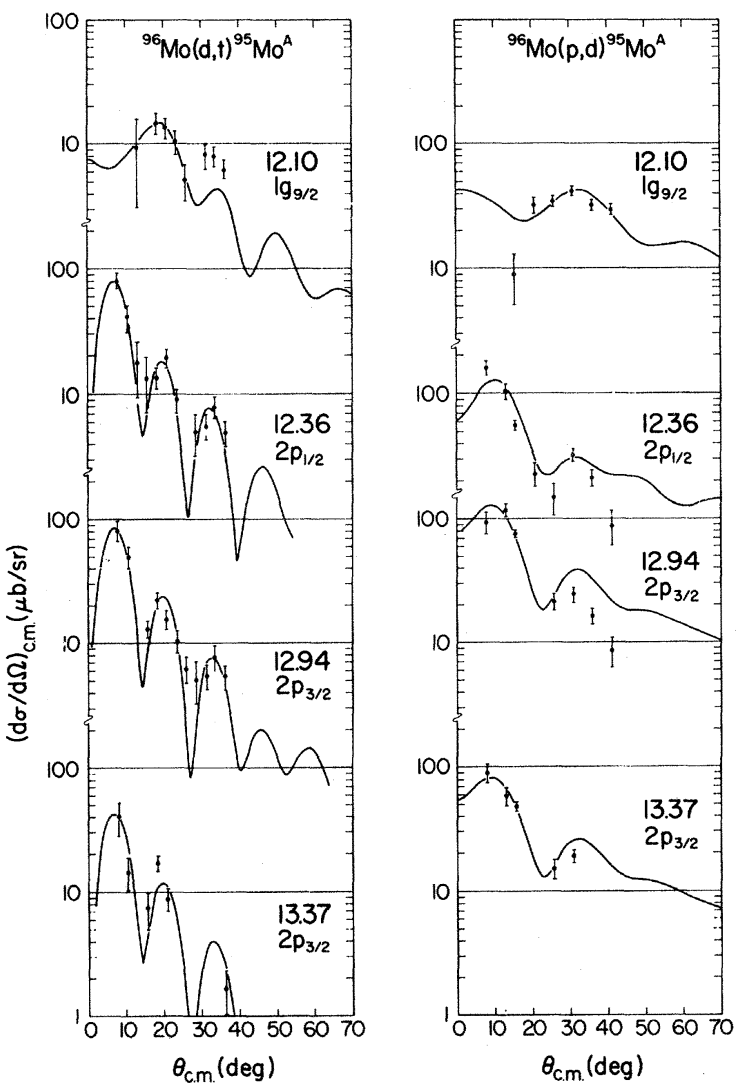

FIG. 7. Angular distributions for $1 g$ and $2 p$ IAS excited in the $(d, t)$ and $(p, d)$ reactions on ${ }^{96} \mathrm{Mo}$. Excitation energies are in $\mathrm{MeV}$, and the curves are DWBA predictions.
The measured Coulomb energies for $1 g_{9 / 2}$ levels are always 20-60 keV lower than for $2 p$ levels. A shift in this direction is to be expected from the Coulomb energy differences between $1 g$ and $2 p$ protons and from binding energy effects. ${ }^{14}$

\section{Spectroscopic Factors}

According to the sum rules for single-nucleontransfer reactions, ${ }^{19}$ the IAS spectroscopic factors are related to those for proton pickup to the parent states by

$$
2 T C^{2} S_{n}=C^{2} S_{p},
$$

where $T \equiv T_{>}$. These quantities are listed in Table II and graphically summarized in Figs. 10 and 11.

The $(p, d)$ spectroscopic factors for $l=4$ and $l=1$ IAS average about $10 \%$ larger than those obtained from the $(d, t)$ reaction. However, the ratios $R_{n n}$ $\equiv C^{2} S_{n}(p, d) / C^{2} S_{n}(d, t)$, which might be compared for levels of the same $J^{\pi}$, are somewhat inconsistent (see Fig. 10). Anomalies are observed in the $2 p_{1 / 2}$ and $1 g_{9 / 2}$ ratios for ${ }^{93} \mathrm{Mo}$, which appear to arise from an unusual $1 g_{9 / 2}-2 p_{1 / 2}$ strength distribution in the $(d, t)$ reaction (Table II). The cross sections for both reactions were extracted
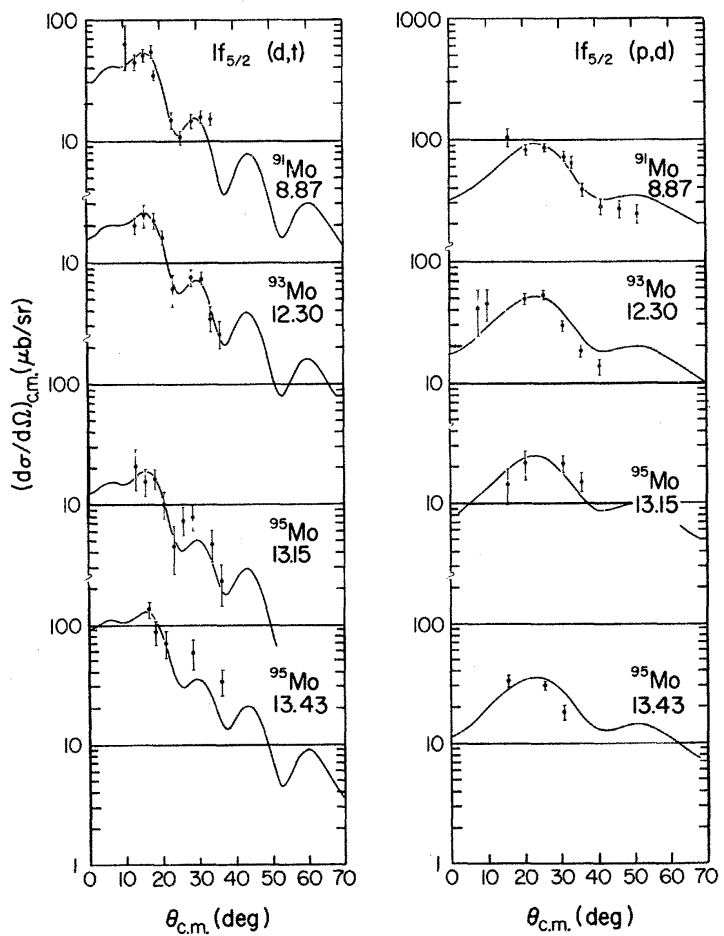

FIG. 8. Angular distributions for $1 f_{5 / 2}$ IAS excited in the $(d, t)$ and $(p, d)$ reactions on ${ }^{92,}{ }^{94,96}$ Mo. Excitation energies are in MeV and the curves are DWBA predictions. 
for the partially resolved doublet at $10.9 \mathrm{MeV}$ excitation with a peak-fitting code. The entire strength of the doublet was assigned to the $\frac{1}{2}^{-}$component at the smallest angle measured for the $(d, t)$ reaction (see Fig. 6), so no significant increase in $2 p_{1 / 2}$ strength is attainable. The $2 p_{1 / 2}$ and $1 g_{9 / 2}$ $(p, d)$ distributions appear to be somewhat distorted, probably because of uncertainties in the peak-fitting procedure. However, if the $2 p_{1 / 2}$ ratio for ${ }^{93} \mathrm{Mo}$ is reduced to agree with those for ${ }^{91} \mathrm{Mo}$ and ${ }^{95} \mathrm{Mo}$ by renormalizing the DWBA curve for the $(p, d)$ reaction, the data points at the two smallest angles would miss the curve by more than two standard deviations. Also, as shown in Fig. 10, $R_{n n}$ for the $\frac{3}{2}^{-}$level of lowest energy is consistently lower than for the second $\frac{3}{2}^{-}$level. Most of these ratios have assigned uncertainties of about $\pm 15 \%$, which reflects a conservative estimate of possible inconsistencies in normalizing the DWBA curves to the data, and no evidence for large discrepancies among $T_{<}$states in these nu$\mathrm{clei}^{16}$ has been observed. The ratio of summed IAS strengths for the $(p, d)$ and $(d, t)$ reactions is constant to within $<10 \%$ from nucleus to nucleus [curve (d) in Fig. 11].

The $\left(d,{ }^{3} \mathrm{He}\right)$ spectroscopic factors summed over

TABLE II. Summary of results for neutron-pickup reactions to IAS in Mo isotopes .

\begin{tabular}{|c|c|c|c|c|c|c|c|c|c|}
\hline$A, T$ & $\begin{array}{l}\text { Mo*a } \\
(\mathrm{MeV})\end{array}$ & $\begin{array}{l}\mathrm{Nb} * \mathrm{~b} \\
(\mathrm{MeV})\end{array}$ & $\begin{array}{c}\Delta_{c}{ }^{\mathrm{c}} \\
(\mathrm{MeV} \pm \mathrm{keV})\end{array}$ & $J^{\pi \mathrm{b}}$ & $\begin{array}{c}\sigma_{\max }{ }^{d} \\
(\mu \mathrm{b} / \mathrm{sr} r)\end{array}$ & $(p, d)$ & $\begin{array}{c}2 T C^{2} S_{n} \\
\quad(d, t)\end{array}$ & $\left({ }^{3} \mathrm{He}, \alpha\right)^{\mathrm{e}}$ & $\begin{array}{c}C^{2} S_{p}{ }^{\mathrm{f}} \\
\left(d,{ }^{3} \mathrm{He}\right)\end{array}$ \\
\hline \multirow[t]{5}{*}{$91, \frac{9}{2}$} & 6.99 & 0 & $12.21 \pm 35$ & $\frac{g}{2}^{+}$ & $92 \pm 5$ & 2.48 & 2.23 & 2.29 & 2.6 \\
\hline & 7.12 & 0.10 & $12.24 \pm 35$ & $\frac{1-}{2}$ & $380 \pm 30$ & 1.59 & 1.77 & 1.58 & 1.4 \\
\hline & 8.34 & 1.31 & $12.25 \pm 35$ & $\left(\frac{3}{2}\right)$ & $295 \pm 30$ & 1.37 & 1.30 & 1.43 & 1.1 \\
\hline & 8.66 & $1.61 \mathrm{~g}$ & $12.27 \pm 35$ & $\left(\frac{3}{2}^{-}\right)$ & $610 \pm 40$ & 3.01 & 2.29 & 2.20 & 2.4 \\
\hline & 8.87 & 1.85 & $12.24 \pm 50$ & $\left(\frac{5}{2}^{-}\right)$ & $93 \pm 10$ & 3.70 & 5.74 & 7.41 & 4.3 \\
\hline \multirow[t]{5}{*}{$93, \frac{11}{2}$} & 10.89 & 0 & $12.09 \pm 30$ & $\frac{g}{2}^{+}$ & $57 \pm 6$ & 2.25 & 2.77 & & $(2.9)$ \\
\hline & 10.94 & 0.03 & $12.11 \pm 30$ & $\frac{1-}{2}$ & $190 \pm 10$ & 1.17 & 0.87 & & (1.6) \\
\hline & 11.59 & 0.68 & $12.11 \pm 50$ & $\left(\frac{3-}{2}\right)$ & $170 \pm 15$ & 1.07 & 0.98 & & 1.1 \\
\hline & 12.22 & \multirow{2}{*}{1.32} & \multirow{2}{*}{$(12.14 \pm 50)$} & $\left(\frac{3}{2}^{-}\right)$ & $250 \pm 20$ & 1.73 & 1.21 & & (1.8) \\
\hline & 12.30 & & & $\left(\frac{5-}{2}\right)$ & $55 \pm 5$ & 2.73 & 3.71 & & $(3.2)$ \\
\hline \multirow[t]{6}{*}{$95, \frac{13}{2}$} & 12.10 & 0 & $11.96 \pm 30$ & $\frac{9}{2}^{+}$ & $43 \pm 4$ & 2.25 & 1.86 & 2.81 & 2.9 \\
\hline & 12.36 & 0.23 & $11.99 \pm 30$ & $\frac{1}{2}^{-}$ & $128 \pm 10$ & 1.13 & 1.06 & 1.29 & 1.6 \\
\hline & 12.94 & 0.77 & $12.03 \pm 50$ & $\left(\frac{3^{-}}{2}\right)$ & $128 \pm 10$ & 1.15 & 1.14 & 0.89 & 1.8 \\
\hline & 13.15 & 0.98 & $12.03 \pm 50$ & $\frac{5}{2}$ & $25 \pm 4$ & 1.79 & 3.59 & 2.78 & 2.0 \\
\hline & 13.37 & \multirow[t]{2}{*}{1.22} & \multirow[t]{2}{*}{$(12.04 \pm 50)$} & $\left(\frac{3}{2}^{-}\right)$ & $82 \pm 7$ & 0.78 & 0.67 & & \multirow[t]{2}{*}{$(2.5)$} \\
\hline & 13.43 & & & $\left(\frac{5^{-}}{2}\right)^{h}$ & $35 \pm 5$ & 2.40 & 2.67 & & \\
\hline \multirow[t]{7}{*}{$97, \frac{15}{2}$} & 13.03 & 0 & $11.88 \pm 30$ & $\frac{g}{2}^{+}$ & $31 \pm 3$ & 2.10 & & & 2.1 \\
\hline & 13.79 & 0.74 & $11.90 \pm 30$ & $\frac{1}{2}^{-}$ & $81 \pm 10$ & 1.00 & & & 1.1 \\
\hline & 14.26 & & & & & & & & \\
\hline & 14.30 & \multirow[t]{3}{*}{1.24} & \multirow[t]{3}{*}{$(11.93 \pm 50)$} & $\left(\frac{3}{2}^{-}\right)$ & $86 \pm 15$ & 1.09 & & & \multirow[t]{3}{*}{2.6} \\
\hline & 14.34 & & & $\left(\frac{3^{-}}{2}\right)$ & $75 \pm 15$ & 0.94 & & & \\
\hline & 14.38 & & & & & & & & \\
\hline & 14.50 & 1.43 & $11.92 \pm 30$ & $\left(\frac{5^{-}}{2}\right)$ & $20 \pm 4$ & 1.74 & & & 2.3 \\
\hline
\end{tabular}

a $\pm 30 \mathrm{keV}$.

${ }^{b}$ See Ref. 5 and references therein.

${ }^{c}$ Values in parentheses are averages for unresolved-parent-state groups.

d Value at forward maximum of DWBA curve after normalization to data from the $(p, d)$ reaction.

e Normalized to give a summed strength for $1 \mathrm{~g}$ and $2 p$ levels equal to that from the $\left(d,{ }^{3} \mathrm{He}\right)$ reaction $(7.5)$ for $A=91$.

f FRNL results of Ref. 5, renormalized to correspond to $N=2.30$ (see text).

g See Ref. 18.

${ }^{\mathrm{h}}$ Assigned in present work. 
$1 g$ and $2 p$ orbitals are essentially constant for $A=91,93$, and 95 [curve (a), Fig. 11], as expected. The value reported by OY for ${ }^{97} \mathrm{Nb}$ is somewhat lower. The IAS strength is not constant, however, but decreases with increasing neutron number. For both the $(p, d)$ and $(d, t)$ reactions, the total $(1 g+2 p)$ strength is nearly $45 \%$ lower for $A=95$ than $A=91$. Most of this is due to a decrease in $l=1$ strength which, for the $(p, d)$ reaction, is a factor of 2 between $A=91$ and $A=97$ [curve (b), Fig. 11], and the relative $l=1$ decrease is largest for the $\frac{3}{2}^{-}$levels. The change in $(p, d)$ spectroscopic factors for the $1 g_{9 / 2}$ ground-state analogs is less than $20 \%$ [curve (c)]. The DWBA calculations are insensitive to slight chinges in target mass and, since the binding energies of the transferred neutrons all lie in an interval of 3.5 $\mathrm{MeV}$, it seems unlikely this effect is due to incorrect $Q$ dependence in the calculations. Data were taken at a few angles for the $\left({ }^{3} \mathrm{He}, \alpha\right)$ reaction on ${ }^{92}$ Mo and ${ }^{96}$ Mo as a check of the latter possibility (Fig. 12), since the $Q$ dependence of this reaction is substantially different from that of either the $(p, d)$ or $(d, t)$ reactions. For example, the ratios of the peak DW cross section for the $8.34-\mathrm{MeV}$ level in ${ }^{91} \mathrm{Mo}$ to that for the $12.94-\mathrm{MeV}$ level in ${ }^{95} \mathrm{Mo}$ are $1.52,1.33$, and 1.04 for the $(p, d)$, $(d, t)$, and $\left({ }^{3} \mathrm{He}, \alpha\right)$ reactions, respectively. The relative decrease in measured $l=1$ strength from $A=91$ to 95 is the same for all three reactions to within $10 \%$, which indicates the general trend is independent of the specific features of a given reaction mechanism. There appears to exist no common trend for the $l=4$ strength, or for the $1 f_{5 / 2}$ strength.

\section{Isospin Mixing}

The decrease in IAS hole strength might be explained by considering the mechanism that leads to spreading widths for isobaric-analog resonances (IAR). This subject has been treated in some detail by Mekjian, ${ }^{20}$ who attributes the spreading width to Coulomb mixing of the IAR with the dense spectrum of $T_{<}$states in the region of the IAR. These $T_{<}$states are in turn coupled by nuclear forces to low-lying configuration states and to isovectormonopole states which lie above the IAR in energy. The monopole states are responsible for the dominant contribution to the mixing strength in the $A=90-100$ region. ${ }^{20}$ Single-particle IAR therefore contain $T>$ and $T_{<}$components, both of which are likely to decay by proton emission and have comparable widths.

It seems reasonable that isospin mixing of a similar nature occurs for hole-state analogs, such as those observed in the present work. However,

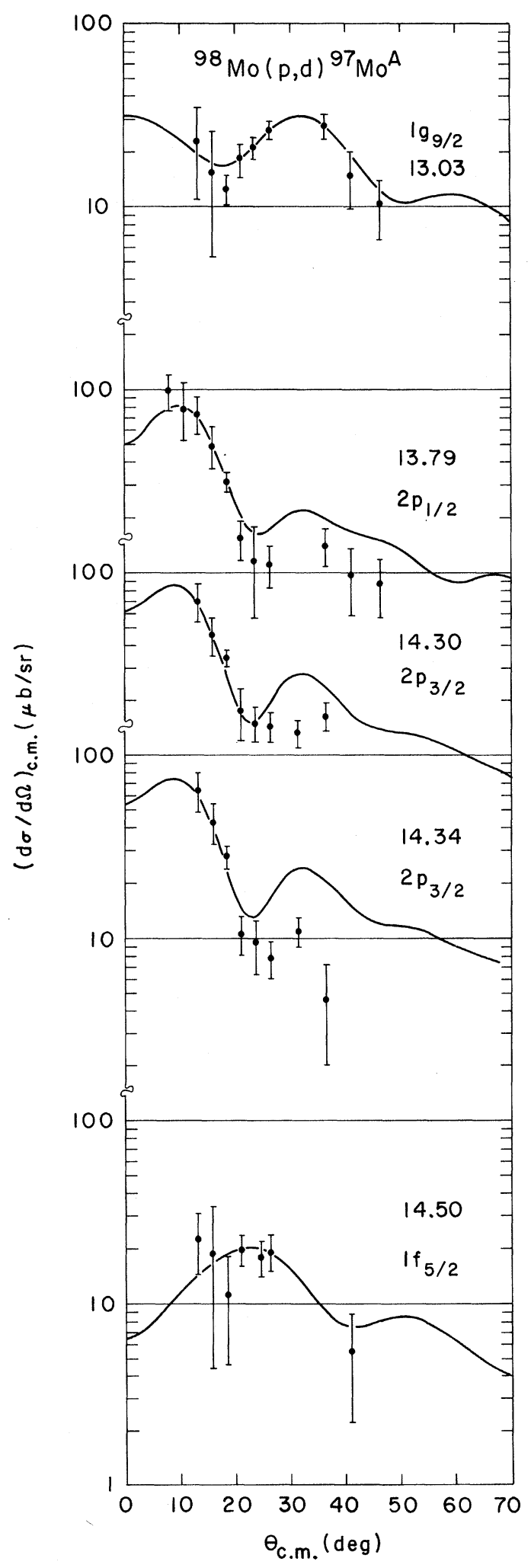

FIG. 9. Angular distributions for IAS excited in the ${ }^{98} \mathrm{Mo}(p, d){ }^{97} \mathrm{Mo}^{A}$ reaction. Excitation energies are in $\mathrm{MeV}$, and the curves are DWBA predictions. 
one must then consider the decay modes of $T>$ and $T<$ hole states whose excitation energies are above the threshold for particle emission. It would seem that the decay of $T_{<}$components would be dominated by neutron emission if that channel is open, and that such states would be quite broad and probably not observable in our spectra due to the high background. The $T_{<}$components in the IAS region are neutron bound in the present work for $A=91$, and become increasingly unbound for $A \geqslant 93$. The $T_{>}$ components are restricted to proton or $\gamma$ decay in all cases by isospin conservation and therefore should be relatively narrow. It seems reasonable, then, that the strength which appears to be missing from the IAS groups in our spectra is contained in broad, unobserved $T_{<}$levels which undergo neutron decay. Also, a decrease in measured IAS hole strength with increasing $A$ is consistent with the IAS moving closer to the monopole with increasing neutron number. ${ }^{21}$ A similar decrease in strength was observed by Sherr et al. ${ }^{22}$ who studied $1 f_{7 / 2}$ IAS via the $(p, d)$ reaction in the $\mathrm{Ni}$ region, but this decrease was partially attributed ${ }^{23}$ to experimental uncertainties. Unusually low spectroscopic factors were obtained ${ }^{23}$ for the IAS in ${ }^{57} \mathrm{Fe},{ }^{61} \mathrm{Ni}$, and ${ }^{59} \mathrm{Ni}$, the first two of which are above the neutron emission threshold. Our results suggest the possibility of an $l=1$ hole-strength minimum around $A=95-97$ [curve (b), Fig. 11], which could correspond to a maximum in the mix-

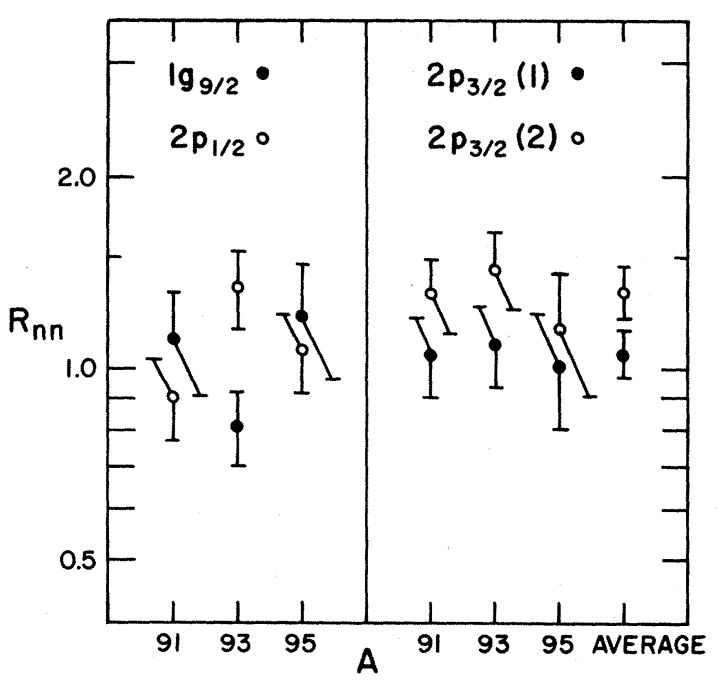

FIG. 10. Spectroscopic factor ratios $R_{n n} \equiv C^{2} S_{n}(p, d) /$ $C^{2} S_{n}(d, t)$ versus mass number. First (lowest excitation) and second $2 p_{3 / 2}$ levels are denoted by (1) and (2), respectively. Weighted averages of $R_{n n}$ are also shown for $2 p_{3 / 2}(1)$ and $2 p_{3 / 2}$ (2). The errors include possible inconsistencies in normalizing DWBA curves to the data and uncertainties in relative normalizations for targets of different $A$. ing strength for these states. Mekjian ${ }^{20}$ has predicted a slight maximum in the IAR monopolemixing strength around $A=90$.

The spins and parities of the dominant monopole contributions in these nuclei are not clear, since many configurations are possible. ${ }^{21}$ The fact that the depletion of spectroscopic strength is concentrated in $l=1$ transitions seems to imply monopole $J^{\pi}$ values of $\frac{1}{2}^{-}$or $\frac{3}{2}^{-}$may be dominant over $\frac{9}{2}^{+}$, or that the $\frac{9}{2}^{+} T_{<}$states which mix with the IAS are sufficiently narrow to be included in our extracted peak yields. The second possibility seems reasonable if one considers the simplest mechanism for the particle decay of a hole state, i.e., a nuclear Auger process whereby the hole is filled by one particle and all the energy is transferred to a sec-

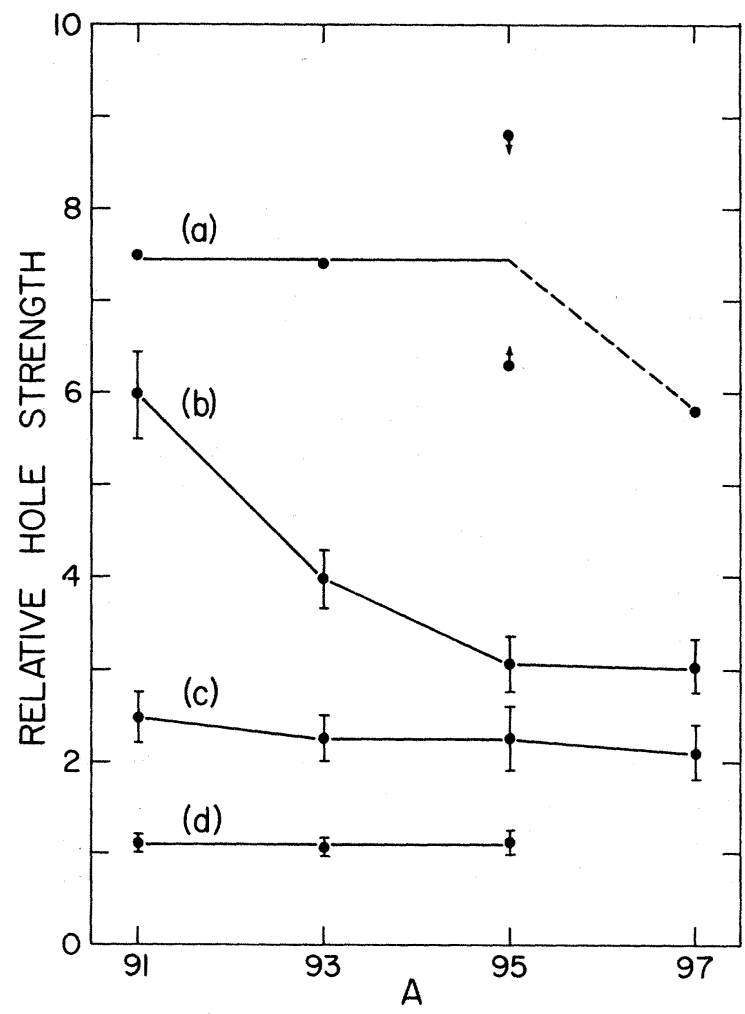

FIG. 11. Spectroscopic strengths and ratios versus mass number. Curve (a) is the summed $(1 g+2 p)$ protonhole strength measured by Ref. 5 (see text regarding normalization). The values for $A=95$ are upper and lower limits, which depend on whether all or none of their unresolved $\left(\frac{3}{2}^{-}+\frac{5}{2}^{-}\right)$doublet is assigned $l=1$ (see also Table II). Curve (b) is $2 T C^{2} S_{n}(p, d)$, summed over the three $l=1$ IAS observed in each nucleus. Curve (c) is $2 T C^{2} S_{n}(p, d)$ for the $g_{9 / 2}$ IAS. Curve (d) is $R_{s} \equiv \Sigma C^{2} S_{n}(p, d) /$ $\Sigma C^{2} S_{n}(d, t)$, where the sums are over the $l=1$ and $l=4$ IAS. The errors for curves (b), (c), (d) include possible inconsistencies in normalizing DWBA curves to the data and uncertainties in relative normalizations for targets of different $A$. 

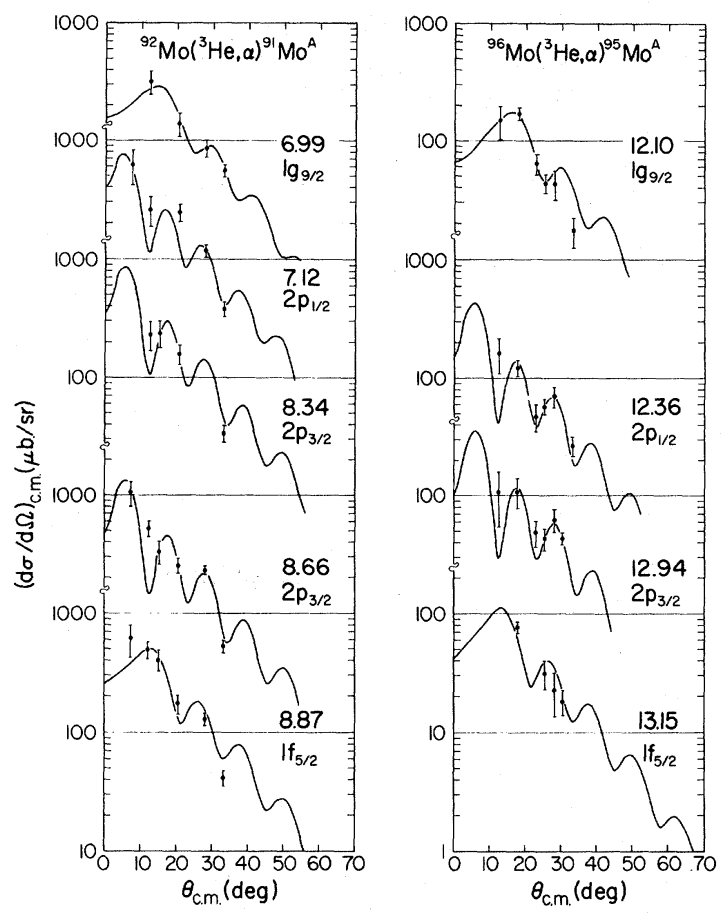

FIG. 12. Angular distributions for IAS excited in the $\left({ }^{3} \mathrm{He}, \alpha\right)$ reaction on ${ }^{92} \mathrm{Mo}$ and ${ }^{96} \mathrm{Mo}$. Excitation energies are in $\mathrm{MeV}$, and the curves are DWBA predictions.

ond particle which is subsequently emitted. This simple process would imply neutron decay to eveneven ground states $\left(J^{\pi}=0^{+}\right)$for the isotopes considered here. If the hole is initially in the $(n, l, j)$ orbit, the emitted neutron would have to decay from the $(n+1, l, j)$ orbit in order to preserve $J^{\pi}$ in the compound system. From shell-model considerations, it appears the $3 p$ orbits are much more accessible than $2 g_{9 / 2}$ orbits at the excitation energies involved here. Thus, the $1 g_{9 / 2}$ hole states may instead prefer to decay by $\gamma$ emission, or by proton emission from one of the $3 s-2 d$ shell orbits, many of which can couple to the nonzero, odd-odd
$\mathrm{Nb}$ core to maintain a total $J^{\pi}$ of $\frac{9}{2}^{+}$. Such states would have considerably smaller widths than neutron-decaying states due to the Coulomb barrier, and may be included in our peak yields for the IAS. It is still not clear, however, whether the observed $l$ dependence is due to the nature of the $T_{<}$state decay, or a selectivity in the mixing strength itself which in turn could arise from a $J^{\pi}$ preference for isovector-monopole states.

\section{SUMMARY AND CONCLUSIONS}

The study of the IAS excited in neutron-pickup reactions on Mo isotopes has revealed a pronounced decrease in hole strength with increasing mass number, as well as fluctuations in the ratios $C^{2} S_{n}(p, d) / C^{2} S_{n}(d, t)$. The decrease in strength might be qualitatively explained by assuming that isospin mixing occurs and that the $T_{<}$states involved in the mixing are very broad and decay primarily by neutron emission. The decrease is concentrated in the $l=1 \mathrm{IAS}$, which suggests an $l$ dependence in the mixing strength or in the decay modes of the $T_{<}$states involved in the mixing. A study of the decay products of the IAS in these nuclei should prove interesting.

There may be a saturation or a maximum in the mixing strength in the $A=95-97$ region of these isotopes (Fig. 11), and we plan to investigate this further with the ${ }^{100} \mathrm{Mo}\left(d,{ }^{3} \mathrm{He}\right){ }^{99} \mathrm{Nb}$ and ${ }^{100} \mathrm{Mo}(p, d)$ ${ }^{99} \mathrm{Mo}^{A}$ reactions. In addition to providing information on Coulomb energies and isospin-mixing strengths, studies of this nature might yield information regarding the spins and parities of monopole excitations.

\section{AC'rNOWLEDGMENTS}

The authors wish to thank L. Wilhoit, S. Self, R. Weber, and Dr. L. G. Keller for their assistance in data acquisition and analysis.
*Research supported in part by the U. S. Atomic Energy Commission and the National Science Foundation. $\dagger$ Present address: Department of Chemistry, Columbia University, New York, New York 10027.

${ }^{1}$ See, e.g., D. Robson, J. D. Fox, P. Richard, and C. F. Moore, Phys. Letters 18, 86 (1965).

${ }^{2}$ R. L. McGrath, N. Cue, W. R. Hering, L. L. Lee, Jr., B. L. Liebler, and Z. Vager, Phys. Rev. Letters 25, $682(1970)$.

${ }^{3}$ S. A. A. Zaidi, C. L. Hollas, J. L. Horton, P. J. Riley, J. L. C. Ford, Jr., and C. M. Jones, Phys. Rev. Letters 25, 1503 (1970).

${ }^{4}$ R. L. Kozub and D. H. Youngblood, Phys. Rev. C $\underline{4}$, 535 (1971).
${ }^{5} \mathrm{H}$. Ohnuma and J. L. Yntema, Phys. Rev. 176, 1416 (1968); 178, 1654 (1969).

${ }^{6}$ R. L. Kozub and D. H. Youngblood, Phys. Rev. Letters 28,1529 (1972).

${ }^{7}$ Received from P. D. Kunz, University of Colorado.

${ }^{8}$ F. D. Becchetti, Jr., and G. W. Greenlees, Phys. Rev. 182,1190 (1969).

${ }^{9}$ C. M. Perey and F. G. Perey, Phys. Rev. 152,923 (1966).

${ }^{10}$ E. R. Flynn, D. D. Armstrong, J. G. Berry, and A. G. Blair, Phys. Rev. 182, 1113 (1969).

${ }^{11}$ C. M. Fou and R. W. Zurmühle, Phys. Rev. 176, 1339 (1968).

${ }^{12}$ W. H. Smith, Phys. Rev. Letters 23, 1045 (1969); and 
private communication.

${ }^{13}$ W. J. Thompson and W. R. Hering, Phys. Rev. Letters $24,272(1970)$.

${ }^{14} \mathrm{~J}$. A. Nolen, Jr., and J. P. Schiffer, Ann. Rev. Nucl. Sci. 19, 471 (1969), and references therein.

${ }^{15} \mathrm{~J}$. H. E. Mattauch, W. Thiele, and A. H. Wapstra, Nucl. Phys. 67, 1 (1965).

${ }^{16}$ R. L. Kozub and D. H. Youngblood, preceding paper, Phys. Rev. C 7 , 404 (1973).

${ }^{17}$ F. Rauch, $\bar{Z}$. Physik 243, 105 (1971).

\author{
${ }^{18} \mathrm{~K}$. Hesse and E. Finckh, Nucl. Phys. A141, 417 (1970). \\ ${ }^{19} \mathrm{~J}$. P. Schiffer, in Isospin in Nuclear Physics, edited \\ by D. H. Wilkinson (North-Holland, Amsterdam, 1969), \\ Chap. 13. \\ ${ }^{20}$ A. Z. Mekjian, Phys. Rev. Letters 25, 888 (1970). \\ ${ }^{21}$ N. Auerbach, Nucl. Phys. A182, 247 (1972). \\ ${ }^{22}$ R. Sherr, B. F. Bayman, E. Rost, M. E. Rickey, and \\ C. G. Hoot, Phys. Rev. 139, B1272 (1965). \\ ${ }^{23}$ E. Rost, Phys. Rev. 154, 994 (1967).
}

\author{
Study of High -Lying States in ${ }^{179} \mathrm{Hf}$ and ${ }^{183,184} \mathrm{~W}$ with the $(n, \gamma)$ Reaction \\ R. F. Casten and W. R. Kane \\ Brookhaven National Laboratory, Upton, New York 11973 \\ (Received 21 July 1972)
}

\begin{abstract}
The nuclei ${ }^{179} \mathrm{Hf},{ }^{183,184} \mathrm{~W}$ were studied with the $(n, \gamma)$ reaction for neutrons of several resonant energies. High- and low-energy $\gamma$ radiation following resonant capture was observed with $\mathrm{Ge}(\mathrm{Li})$ detectors. Level schemes were constructed from these data in conjunction with previous information from charged-particle reactions and decay studies. Some discrepancies among previous studies were clarified and a large number of spin assignments suggested. From these and data from the $(d, p)$ reaction an analysis is made of the fractionation and distribution of the single-particle Nilsson strength in the energy region 1.3-2.3 MeV. It is found that the Nilsson model breaks down rather suddenly above the vibrational energy: A much larger than expected number of rotational bands are observed and the $(d, p)$ strength is severely fragmented. This mixing appears to be larger in ${ }^{183} \mathrm{~W}$ than in ${ }^{179} \mathrm{Hf}$ and still larger in ${ }^{184} \mathrm{~W}$. The data for $2^{+}$and $1^{+}$states in ${ }^{184} \mathrm{~W}$ are compared with recent random-phaseapproximation calculations: Again, much more configuration mixing is observed than is calculated and significant $(d, p)$ strength occurs lower than predicted in the spectrum.
\end{abstract}

\section{INTRODUCTION}

In its description of the low-excitation-energy region of heavy deformed nuclei the Nilsson model ${ }^{1}$ has been remarkably successful. ${ }^{2-5}$ Through it, an immense body of data from charged-particle reactions and electromagnetic decay studies has been interpreted and correlated.

In odd nuclei (for example the $150 \leqslant A \leqslant 190$ deformed region) it has been possible to interpret most states up to $\sim 1200 \mathrm{keV}$ in terms of simple one-quasiparticle-proton or -neutron excitations. The Nilsson model provides simple predictions for one-nucleon-transfer-reaction cross sections that are generally in very good agreement with experimental results. It is often necessary to incorporate an analysis of the effects of Coriolis or $\Delta N=2$ mixing or occasionally of hexadecapole deformations on these cross sections, but numerous examples exist ${ }^{6-8}$ for which, once done, the absolute values and systematics of stripping- and picking-reaction cross sections are excellently reproduced. Similarly the model establishes many elec- tromagnetic and $\beta$-decay selection rules and estimates of matrix elements that have been found to be in satisfactory agreement with experiment. In turn, as with the charged-particle data, these have allowed the assignment of most low-lying levels to the excitation of various Nilsson orbitals and to the rotational bands built upon them.

At about $1 \mathrm{MeV}$ (in the rare-earth region) vibra tional excitations can occur and the lowest lying of these ( $\gamma$ and $\beta$ vibrations) have been reasonably successfully interpreted both microscopically and macroscopically..$^{9-12}$ These excitations are obtained theoretically ${ }^{9,11}$ typically as the lowest roots of secular equations in the random-phase approximation. Higher roots should also exist but have seldom been unambiguously identified experimentally.

In fact, at energies above the first vibrational excitations the entire model appears to break down. It rapidly becomes difficult or impossible to as sign Nilsson orbitals to rotational bands (or even to locate well-behaved bands) above $\sim 1500 \mathrm{keV} .{ }^{13,14}$ Generally, only high-spin levels at these energies 